\title{
EFEITOS FÍSICOS E PSICOSSOCIAIS DA INSUFICIÊNCIA CARDÍACA NA PERCEPÇÃO DA QUALIDADE DE VIDA*
}

\author{
Mailson Marques de Sousa ${ }^{1}$, Jacira dos Santos Oliveira ${ }^{2}$, Maria Julia Guimarães Oliveira Soares ${ }^{3}$, Simone Maria \\ Muniz da Silva Bezerra ${ }^{4}$, Simone Helena dos Santos Oliveira ${ }^{3}$
}

\begin{abstract}
RESUMO: Objetivou-se analisar a qualidade de vida relacionada à saúde de pacientes com insuficiência cardíaca e apresentar proposta de intervenção para as variáveis que mais afetam a percepção da qualidade de vida. Estudo descritivo, transversal, com abordagem quantitativa, com 84 pacientes com insuficiência cardíaca em seguimento ambulatorial realizado no município de João Pessoa, PB, Brasil, no período de janeiro a julho de 2015. Aplicou-se o Minnesota Living With Heart Failure Questionnaire. A média do escore total do Minnesota Living With Heart Failure Questionnaire foi 33,13 $\pm 19,66$ revelando boa qualidade de vida. No entanto, os aspectos físicos e a dificuldade de adesão à dieta foram as variáveis com maior influência negativa na percepção da qualidade de vida. Sugere-se a proposição de estratégias terapêuticas, com foco multidisciplinar para os aspectos físicos e psicossociais mais afetados para minimizar o impacto da insuficiência cardíaca na qualidade de vida relacionada à saúde.
\end{abstract}

DESCRITORES: Doenças crônicas; Insuficiência cardíaca; Qualidade de vida; Estilo de vida.

\section{PHYSICAL AND PSYCHOSOCIAL EFFECTS OF HEART FAILURE IN PERCEIVED QUALITY OF LIFE}

\begin{abstract}
The objective was to analyze the health-related quality of life of heart failure patients and to present an intervention proposal for the variables that most affect the perceived quality of life. Descriptive, cross-sectional study with a quantitative approach, involving 84 heart failure patients under outpatient follow-up, undertaken in the city of João Pessoa, PB, Brazil, between January and July 2015. The Minnesota Living Heart Failure Questionnaire was applied. The average total score on the Minnesota Living Heart Failure Questionnaire was $33.13 \pm 19.66$, revealing a good quality of life. The physical aspects and difficulty to comply with the diet were the variables that most negatively influenced the perceived quality of life. Therapeutic strategies should be proposed with a multidisciplinary focus for the most affected physical and psychosocial aspects, with a view to minimizing the impact of the heart failure on the health-related quality of life.
\end{abstract}

DESCRIPTORS: Chronic disease; Heart failure; Quality of life; Life style.

\section{EFECTOS FÍSICOS Y PSICOSOCIALES DE LA INSUFICIENCIA CARDÍACA EN LA CALIDAD DE VIDA PERCIBIDA}

RESUMEN: El objetivo fue analizar la calidad de vida relacionada a la salud de pacientes con insuficiencia cardíaca y presentar propuesta de intervención para las variables que más influyen en la calidad de vida percibida. Estudio descriptivo, trasversal, con aproximación cuantitativa, con 84 pacientes con insuficiencia cardíaca en seguimiento ambulatorio, desarrollado en el municipio de João Pessoa, PB, Brasil, en el período de enero a julio del 2015. Fue aplicado el Minnesota Living Heart Failure Questionnaire. El promedio del score total del Minnesota Living Heart Failure Questionnaire correspondió a 33,13 $\pm 19,66$ revelando buena calidad de vida. Sin embargo, los aspectos físicos y la dificultad de adhesión a la dieta fueron las variables con mayor influencia negativa en la calidad de vida percibida. Se sugiere proponer estrategias terapéuticas con enfoque multidisciplinario para los aspectos físicos y psicosociales más afectados, con vistas a minimizar el impacto de la insuficiencia cardíaca en la calidad de vida relacionada a la salud. DESCRIPTORES: Enfermedad crónica; Insuficiencia cardíaca; Calidad de vida; Estilo de vida.

\footnotetext{
*Artigo extraído da dissertação intitulada: “Avaliação da qualidade de vida relacionada à saúde de pacientes com insuficiência cardíaca". Universidade Federal da Paraíba, 2016.
}

${ }^{1}$ Enfermeiro. Doutorando em Enfermagem. Universidade Federal da Paraíba. João Pessoa, PB, Brasil.

${ }^{2}$ Enfermeira. Doutora em Enfermagem. Docente de Enfermagem da Universidade Federal da Paraíba. João Pessoa, PB, Brasil. ${ }^{3}$ Enfermeira. Doutora em Enfermagem. Docente do Programa de Pós-Graduação em Enfermagem da Universidade Federal da Paraíba. João Pessoa, PB, Brasil.

${ }^{4}$ Enfermeira. Doutora em Ciências. Docente do Programa de Pós-Graduação em Enfermagem da Universidade de Pernambuco. Recife, PE, Brasil.

Autor Correspondente:

Mailson Marques de Sousa

Universidade Federal da Paraíba

R. Othília Barros de Medeiros, 156 - 58037-710 - João Pessoa, PB, Brasil

E-mail:mailson725@gmail.com
Recebido: $15 / 12 / 2016$ Finalizado: $22 / 05 / 2017$ 


\section{INTRODUÇÃO}

A insuficiência cardíaca (IC) é uma síndrome crônica que se manifesta pela incapacidade do coração em encher-se ou ejetar sangue para atender as necessidades metabólicas e teciduais do organismo. A IC é ocasionada por desordens estruturais ou funcionais, sendo considerada um grave e crescente problema de saúde pública, acarretando elevados custos socioeconômicos ${ }^{(1)}$.

Apesar dos avanços no tratamento e manejo clínico, a IC apresenta altas taxas de mortalidade e morbidade, sendo a primeira causa de hospitalização em idosos nos países ocidentais ${ }^{(2)}$. Estimativas apontam que cerca de 6,5 milhões de pessoas na Europa, cinco milhões de pessoas nos Estados Unidos e 2,5 milhões depessoas no Japão, sofrem de IC, e anualmente cerca de um milhão de novos casos são diagnosticados no cenário mundial ${ }^{(3)}$. No Brasil, aproximadamente 6,4 milhões de pessoas apresentam a enfermidade, sendo a primeira causa de hospitalizações por doença cardiovascular ${ }^{(4)}$.

Evidências apontam a baixa adesão ao tratamento, o desconhecimento da síndrome, a falta de apoio social e o seguimento inadequado, como os principais fatores de descompensação da enfermidade ${ }^{(3)}$. Nesse contexto, tem-se buscado alternativas para reduzir as taxas de mortalidade e reinternações hospitalares, de modo a favorecer a diminuição dos custos em saúde e minimizar o impacto da insuficiência cardíaca na qualidade de vida (QV) dos seus portadores.

A QV tem sido objeto de estudo com a finalidade de promover a tomada de decisão, identificar a melhor conduta terapêutica na prática clínica e auxiliar no desenvolvimento de programas de promoção e reabilitação a saúde, com foco no cuidado integral e humanizado ${ }^{(5)}$. A qualidade de vida relacionada à saúde (QVRS) é apreendida como impacto dos sintomas, da incapacidade ou limitações de uma enfermidade sobre a percepção de bem-estar ${ }^{(6)}$.

No âmbito da insuficiência cardíaca, as pessoas acometidas comparadas à população geral, apresentam QVRS diminuída em virtude da redução da capacidade de realizar atividades da vida diária, ocasionadas pelos sintomas progressivos da doença como dispneia, fadiga, insônia, perda de apetite e diminuição do convívio social ${ }^{(2,4)}$.

No Brasil, são poucos os estudos que avaliam a QVRS em pacientes com IC, sendo as regiões Sul e Sudeste os principais cenários de investigação. Portanto, mostra-se relevante a proposição de novos estudos nas diferentes regiões do país, tendo em vista a pluralidade cultural e dos hábitos da vida diária que podem influenciar a enfermidade, permitindo assim conhecer a QVRS desses pacientes e identificar as dimensões que a comprometem ou a favorecem. Estas informações podem subsidiar a proposição de estratégias consonantes às realidades identificadas, com a finalidade de contribuir para melhoria da QV desses pacientes.

Diante do exposto, os objetivos do estudo foram: determinar o escore de qualidade de vida relacionada à saúde de pacientes com insuficiência cardíaca, identificar os escores médios das variáveis que compõem as dimensões do Minnesota Living With Heart Failure Questionnaire e elaborar proposta de intervenção para as variáveis que influenciam negativamente a percepção da qualidade de vida relacionada à saúde.

\section{MÉTODO}

Estudo descritivo, transversal, com abordagem quantitativa, realizado em duas instituições hospitalares públicas do município de João Pessoa, Paraíba, Brasil, no período de janeiro a julho de 2015. A amostra, não probabilística e consecutiva, foi constituída de 84 pacientes com IC, de ambos os sexos.

Os critérios de inclusão foram pacientes com idade $\geq 18$ anos, em seguimento ambulatorial durante o período de coleta de dados. Foram excluídos pacientes com câncer, doenças pulmonares, com barreiras de comunicação ou os que apresentassem desconforto clínico, como dispneia e fadiga durante a entrevista.

A coleta de dados ocorreu nos dias agendados para consulta ambulatorial, antes ou após a consulta 
médica, em local reservado, por meio de entrevista individual. Para caracterização sociodemográfica e clínica, registraram-se idade, gênero, situação conjugal, escolaridade, raça, classe funcional da insuficiência cardíaca segundo critérios da New York Heart Association (NYHA), fração de ejeção do ventrículo esquerdo, presença de comorbidades e terapêutica medicamentosa.

Para avaliação da QVRS, foi utilizado o questionário Minnesota Living with Heart Failure Questionnaire (MLHFQ), validado para língua portuguesa do Brasil( ${ }^{(7)}$. O MLHFQ é composto de 21 questões, apresentadas na forma de escalas do tipo Likert, com pontuações que variam de 0 (não) a 5 (demais), onde 0 representa "sem limitações" e 5 "limitação máxima". Escores mais altos indicam pior QV e escores mais baixos melhor $\mathrm{QV}^{(7)}$.

Os dados foram analisados usando o software Statistical Package for Social Science (SPSS), versão 21.0. Todas as variáveis foram submetidas à análise descritiva. A confiabilidade das medidas de qualidade de vida foi analisada pela consistência interna das dimensões, utilizando-se o coeficiente de alfa de Cronbach (valores $\geq 0,70$ foram considerados satisfatórios).

O estudo foi aprovado pelo Comitê de Ética em Pesquisa do Hospital Universitário Lauro Wanderley, conforme parecer $n^{\circ}$ 919.725/2014 respeitando as exigências formais contidas nas normas nacionais e internacionais regulamentadoras de pesquisas envolvendo seres humanos.

\section{- RESULTADOS}

A amostra foi constituída de 84 pacientes com IC, com média de idade de 58,82 12,78 anos, variação entre 23 e 86 anos. No que diz respeito ao perfil sociodemográfico, houve distribuição equilibrada entre os sexos, $(n=45,53,6 \%)$ pertenciam ao sexo feminino, $(n=42,50 \%)$ declaram-se da raça branca, $(n=57,67,8 \%)$ era casada ou vivia em união estável. Na amostra, sobressaiu o baixo nível educacional, com $(n=47,56 \%)$ apresentando ensino fundamental incompleto ou completo e $(n=18,21,4 \%)$ analfabetos. A aposentadoria $(n=49,58,3 \%)$ e $(n=41,48,8 \%)$ a renda familiar de dois salários mínimos foram características presentes da amostra.

Em relação às características clínicas, as comorbidades mais frequentes foram a Hipertensão Arterial Sistêmica $(n=53,63,1 \%)$, seguida do Diabetes Mellitus $(n=11,13,1 \%)$. A etiologia da IC mais evidenciada foi a não isquêmica $(n=61,72,6 \%)$ sobressaíram-se participantes nas classes funcionais I e II ( $n=37,44 \%$; $\mathrm{n}=31,36,9 \%$, respectivamente), segundo os critérios estabelecidos pela NYHA. A média da fração de ejeção do ventrículo esquerdo foi de $50 \pm 14,6$, variando de 19 a $74 \%$. Os medicamentos mais utilizados pelos pacientes e registrados em prontuário foram os diuréticos $(n=44,52,4 \%)$. Quatro pacientes $(4,8 \%)$ faziam uso de marcapasso.

Previamente à análise dos resultados obtidos pelo MLHFQ, foi avaliada a consistência interna do instrumento por meio do alfa de Cronbach (escore total $\mathrm{a}=0,90$ ), revelando a confiabilidade para a amostra pesquisada. O escore total e por dimensão da QVRS foi obtido a partir da soma das médias de cada variável que compõe o instrumento. Cada dimensão e o escore total apresentam variações possíveis, conforme se vê na Tabela 1. Os resultados revelaram que o escore total do MLHFQ foi de $33,13 \pm 19,66$, o que indica uma boa QVRS da amostra estudada.

Tabela 1 - Escores das dimensões do Minnesota Living With Heart Failure Questionnarie. João Pessoa, PB, Brasil, 2015

\begin{tabular}{lcccc} 
Dimensões & Escore \pm desvio padrão & Mediana & Variação observada & Variação possível \\
\hline Física & $15,68 \pm 10,97$ & 13,5 & $0-40$ & $0-40$ \\
\hline Emocional & $7,62 \pm 5,05$ & 7 & $0-20$ & $0-25$ \\
\hline Total & $33,13 \pm 19,66$ & 29 & Apr-79 & $0-105$
\end{tabular}

Com o objetivo de avaliar o comportamento das variáveis e sua influência nas dimensões que compõem o MLHFQ, obteve-se as médias das respostas emitidas pelo conjunto de sujeitos para cada 
questão (Figura 1). Na dimensão física, caminhada e subida de escada difícil, falta de ar e fadiga foram as variáveis com maiores médias, portanto as mais comprometidas. Com relação à dimensão emocional, as variáveis preocupação e sensação de depressão foram as mais afetadas. Para as demais variáveis que compõem o instrumento, a ingestão de alimentos preferidos pelos pacientes em menor quantidade desejada foi a que apresentou a pior média entre todos os itens do MLHFQ.

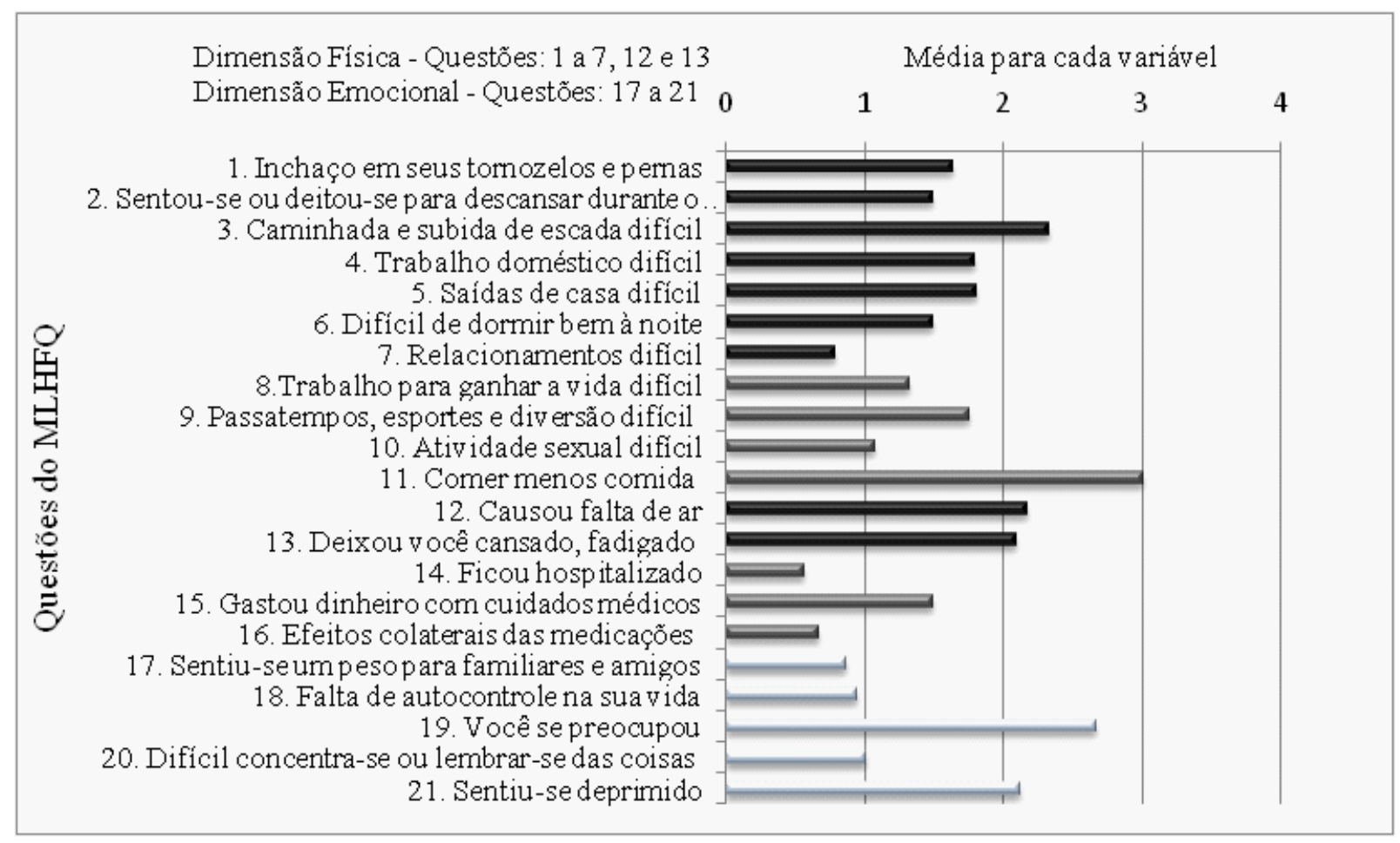

Figura 1 - Média das variáveis que compõem o Minnesota Living With Heart Failure Questionnaire. João Pessoa, 2015

\section{- DISCUSSÃO}

O perfil sociodemográfico caracterizou-se por distribuição equilibrada entre os sexos, com idade média de 58,82 anos, predominando pessoas da raça branca, casadas ou vivendo em união estável, aposentadas e com baixo nível educacional. Com relação à idade, escolaridade e situação profissional, os resultados encontrados se assemelham ao de estudos no cenário nacional ${ }^{(8-9)}$.

Do ponto de vista clínico, Hipertensão Arterial Sistêmica (HAS) e Diabetes Mellitus (DM) foram as comorbidades que se destacaram entre os pesquisados. Esse achado é concordante com estudo desenvolvido na América Latina, que apontou HAS, DM, dislipidemias e obesidade como os principais fatores de risco para o surgimento da insuficiência cardíaca. Portanto, a conjugação dessas comorbidades apresenta sérias implicações cardiocirculatórias e que se não tratadas de forma adequada podem ocasionar a descompensação clínica e agravamento da enfermidade ${ }^{(10)}$.

No que se refere à etiologia, a IC de origem não isquêmica predominou na amostra estudada, embora a literatura internacional aponte a causa isquêmica como a principal. Tal constatação pode estar relacionada à maior frequência da IC de etiologia chagásica e hipertensiva no contexto brasileiro(11).

No que concerne às classes funcionais da insuficiência cardíaca, de acordo com a classificação da NYHA, entre a amostra as classes I e II foram as mais prevalentes, o que demonstra que os pacientes acompanhados apresentavam ausência de sintomatologia ou sintomas desencadeados a partir de esforços.

Por conseguinte, com relação à medida de QVRS avaliada através do MLHFQ, a pontuação média 
mostrou-se baixa para o escore total e para as dimensões física e emocional, já que se situaram abaixo dos valores que se constituem o ponto médio de variação da escala (física: variação 0-40, ponto médio 20; emocional: 0-25, ponto médio 12,5; QV total: 0-105, ponto médio 52,5), evidenciando que, mesmo diante de uma doença crônica com fortes implicações para a saúde global, os pacientes avaliados apresentam boa qualidade de vida relacionada à saúde.

Esses dados nos remetem a reflexões acerca das condições clínicas que caracterizam a amostra, destacando-se a ausência de sintomas ou sintomas desencadeados por esforços físicos, referidos pela maioria dos pesquisados. Neste escopo, destaca-se que todos os pacientes são atendidos ambulatorialmente, o que já indica a estabilização do quadro clínico. Além disso, grande parte da amostra era assídua à consulta médica e estava em seguimento ambulatorial superior a seis meses, e ainda relatava adesão à terapêutica medicamentosa prescrita, o que favorece um menor impacto da IC na percepção da qualidade de vida relacionada à saúde.

Sabe-se que com o aumento progressivo da doença, os sintomas vivenciados pelos pacientes são mais intensos, o que leva à busca por serviços de emergência nos quadros de descompensação clínica. Consoante a esta assertiva, estudo de coorte, realizado com 661 pacientes hospitalizados com IC, seguidos prospectivamente por três anos, constatou pacientes com alta pontuação na dimensão física, relacionada às classes III e IV da NYHA, refletindo uma percepção negativa da QVRS e altas taxas de mortalidade $^{(12)}$. Portanto, é esperado que pacientes em seguimento ambulatorial apresentem melhores escores de QVRS do que aqueles hospitalizados, em decorrência do agravamento dos sintomas da IC que demandam a hospitalização.

Embora os resultados tenham revelado QVRS, procedeu-se a análise do comportamento das variáveis que compõem as dimensões do MLHFQ com intuito de verificar o comportamento de cada variável e sua consequente contribuição para o escore por domínio e total, sendo possível identificar que a dimensão física foi a mais afetada e sofreu influência principalmente da fadiga, dispneia e dificuldades de locomoção.

A fadiga é uma das manifestações mais frequentes em pacientes com IC, assim como a falta de ar e a ortopneia ${ }^{(13)}$. Sintomas de dispneia, fadiga e edema de membros inferiores foram evidenciados como as principais causas de hospitalização ${ }^{(14)}$. A fadiga é ainda apontada como fator associado a limitações para manutenção de um estilo de vida compatível com senso desejável de autonomia e independência ${ }^{(13)}$. Assim, mostra-se coerente a obtenção de escores médios mais altos para as variáveis que compõem a dimensão física.

$\mathrm{Na}$ avaliação dos itens que compõem a dimensão emocional, a questão referente à sensação de preocupação sobressaiu. A literatura revela que os pacientes necessitam realizar mudanças no estilo de vida para atender ao complexo regime terapêutico da enfermidade. Isso implica alterações na percepção de bem-estar pelo fato da IC ser uma doença crônica, que impõe restrições aos hábitos rotineiros, limitando as atividades da vida diária e refletindo na capacidade de autonomia e independência ${ }^{(15)}$. Entende-se que essas limitações físicas passam a influenciar emocionalmente as pessoas afetadas.

Consonante a esse entendimento, a sensação de depressão está presente na maioria das doenças crônicas, especialmente na IC, por ser uma doença de prognóstico não favorável. As mudanças comportamentais potencializam queixas depressivas em virtude da diminuição no prazer sensorial da alimentação, do convívio social e das atividades preferidas, ocasionando perda de autoestima, exclusão familiar e social(16).

Estudos relatam que o medo de perder o autocontrole e da morte, bem como o isolamento social, são os principais impactos na dimensão emocional desses pacientes ${ }^{(17)}$. Além disso, o gasto com remédios e o uso contínuo de medicações para o controle da doença promovem aumento no orçamento doméstico, uma vez que a IC não goza de estatuto especial como outras doenças, o que pode ser a principal causa de abandono do tratamento, alterando dessa forma a percepção de QVRS ${ }^{(16)}$.

Destaca-se ainda, a questão alusiva a hábitos de consumo alimentar, embora não esteja presente em uma dimensão específica, a mesma compõe a soma do escore total do MLHFQ. Para esta variável, constatou-se a maior média entre as questões do instrumento, relevando a dificuldade da população para aderir ao regime dietético necessário ao controle da doença influenciando negativamente a percepção da QVRS. 
Em pacientes com IC, é fundamental a exclusão de alimentos ricos em sódio e a adição do sódio nos alimentos após a preparação, como forma de evitar a retenção de líquidos. Além disso, é essencial a restrição hídrica e o monitoramento do peso para o reconhecimento de sinais de descompensação clínica ${ }^{(18)}$.

Nesse sentido, mister se faz a contextualização cultural no que tange à culinária local, que é fortemente marcada por alimentos ricos em gordura trans, sódio e carboidratos simples, além disso o avanço das redes de fast-food em decorrência do processo de industrialização, contribui para o aumento do sobrepeso e da desnutrição relacionada à diminuição no consumo de cereais, frutas e legumes, favorecendo dessa forma o surgimento de alterações cardiovasculares ${ }^{(19)}$.

Além disso, a dieta hipossódica apresenta baixa adesão em virtude do modo de preparo e dos condimentos utilizados para acentuar o sabor alimentos. No Brasil, a ingestão diária de cloreto de sódio situa-se em torno de 11,4g/dia, ultrapassando em mais de cinco vezes as necessidades diárias ${ }^{(19)}$. No paciente com IC, a ingestão de sódio deve ser, no máximo, de 2-3g/dia, evitando-se o uso de temperos e refeições industrializadas, devendo a restrição de sódio ser adaptada à situação clínica do paciente ${ }^{(18-19)}$. Assim, torna-se fundamental por parte do profissional de saúde, o conhecimento para orientar o paciente e seus familiares sobre mudanças no estilo de vida necessárias para o cuidado em saúde da pessoa com IC.

Adicionalmente, durante o desenvolvimento da pesquisa foi possível identificar a inexistência de programas de acompanhamento multidisciplinar, tampouco por enfermeiros no planejamento da assistência em saúde aos pacientes com IC acompanhados ambulatorialmente. Embora não se tenha constituído objeto de investigação, esse fato revela fragilidade na assistência prestada e a necessidade da gestão dos serviços de saúde de implementar programas com enfoque multidisciplinar, uma vez que estes visam facilitar a compreensão do paciente em relação à doença, bem como o desenvolvimento de estratégias para melhorar a adesão terapêutica e a gestão do autocuidado ${ }^{(20)}$.

É consenso afirmar que intervenções multidisciplinares em pacientes com IC tem evidenciado redução dos índices de readmissão por descompensação, contribuído para diminuição das taxas de mortalidade e para maior adesão às recomendações terapêuticas em ambiente domiciliar ${ }^{(20)}$.

Destaca-se que a enfermagem ocupa papel importante na equipe multiprofissional, uma vez que possui papel educador a partir de orientações educativas sobre o conhecimento da enfermidade, auxiliando o paciente na implementação de medidas de autocuidado e reconhecimento dos sinais e sintomas de descompensação, visitas domiciliares, além de ajustes na dose de diuréticos prescritos, refletindo dessa forma em uma melhor qualidade de vida relacionada à saúde da população assistida ${ }^{(1)}$.

Nesse sentido, a partir da experiência clínica dos autores e baseados nas evidências científicas da Diretriz Brasileira de Insuficiência Cardíaca Crônica da Sociedade Brasileira de Cardiologia ${ }^{(1)}$, vigente para o manejo de pacientes com IC, foi elaborada proposta de intervenção de acordo com as principais variáveis que afetaram negativamente a percepção da QVRS da amostra investigada, tendo como objetivo nortear e orientar os profissionais a intervir sobre os problemas reais e potencias em saúde, no sentido de promover uma melhor aceitação, entendimento e adesão ao plano terapêutico instituído aos pacientes com IC, concorrendo para a estabilidade clínica da enfermidade e para uma boa QVRS.

Para as variáveis como caminhada, subida de escadas, sugere-se a participação em programas de exercícios físicos diários, evitando períodos de extremo calor e frio, bem como realizar caminhadas leves e de maneira gradual, desde que não causem esforço intenso, com a finalidade de fortalecimento da capacidade funcional e reserva energética, evitando-se episódios de hipertrofia miocárdica. Falta de ar e fadiga, recomenda-se realizar e aumentar gradativamente as atividades da vida diária, propiciando ambiente de repouso arejado. Desse modo é possível identificar as atividades da vida diária que acentuam ou não episódios de dispneia.

Com relação às variáveis sentir-se preocupado e sensação de depressão, a participação em grupos de apoio psicológico e em atividades de relaxamento (musicoterapia, ioga, acupuntura) contribuem para promover a sensação de bem-estar e equilíbrio emocional, motivando-o para adesão à terapêutica.

Sugere-se disponibilizar atendimento nutricional em nível ambulatorial, com acompanhamento 
individualizado e atividades de conscientização em grupo, minimizando os efeitos deletérios desencadeados a partir da ingesta inadequada de nutrientes, ocasionado acúmulo de sódio e líquidos, além de identificar sinais de caquexia cardíaca e retenção hídrica.

Como limitação desse estudo, pode-se apontar o delineamento transversal, que impede a avaliação do comportamento das variáveis ao longo do tempo e a inferência de relações de causalidade entre elas. Desse modo, faz-se necessária a proposição estudos de coortes, comparando subgrupos expostos e não expostos a estratégias terapêuticas claramente planejadas pela equipe multiprofissional, de forma a analisar os benefícios do plano terapêutico implementado, a fim de prevenir a descompensação da doença e preservar a boa qualidade de vida relacionada à saúde, além de aprofundar o perfil sociodemográfico e clínico desses pacientes em diferentes regiões do país.

\section{CONCLUSÃo}

Os resultados permitem concluir que os pacientes do estudo apresentam boa QVRS, o que pode ser atribuído ao fato da totalidade da amostra ser proveniente de atendimento ambulatorial e classificados em classe funcional I e II da NYHA, portanto não inclui pacientes com sintomas mais avançados da IC. Além disso, foi possível identificar que, entre as variáveis que afetaram negativamente a percepção da QVRS, os aspectos físicos e a ingesta de alimentos em menor quantidade sobressaíram.

Por ser a IC uma doença crônica que requer mudanças de hábitos de vida e alimentares, nota-se a necessidade de implementar programas e estratégias terapêuticas como abordagem multiprofissional para minimizar o impacto da IC nos aspectos físicos e na adesão às restrições dietéticas, uma vez que o controle dessas variáveis são essenciais para manutenção da capacidade funcional, enfrentamento e estabilidade clínica da enfermidade.

\section{REFERÊNCIAS}

1. Bocchi EA, Marcondes-Braga FG, Bacal F, Ferraz AS, Albuquerque D, Rodrigues D, et al. Sociedade Brasileira de Cardiologia. Atualização da Diretriz Brasileira de Insuficiência Cardíaca Crônica - 2012. Arq Bras Cardiol. [Internet] 2012;98(1 Suppl 1) [acesso em $01 \mathrm{dez}$ 2015]. Disponível: http://publicacoes.cardiol.br/consenso/2012/ Diretriz\%20IC\%20Cr\%C3\%B4nica.pdf.

2. Farmakis D, Parissis J, Karavidas A, Karvounis C, Triposkiadis F, Filippatos G, et al. In-hospital management of acute heart failure: Practical recommendations and future perspectives. Int J Cardiol. [Internet] 2015;(201) [acesso em 06 de jan 2016] Disponível: http://dx.doi.org/10.1016/j.ijcard.2015.08.030.

3. Boisvert S, Proulx-Belhumeur A, Gonçalves N, Doré M, Francoeur J, Gallani MC. An integrative literature reviews on nursing interventions aimed at increasing self-care among heart failure patients. Rev. Latino-Am. Enfermagem. [Internet] 2015;23(4) [acesso em 05 de nov 2015]. Disponível:http://dx.doi.org/10.1590/0104-1169.0370.2612.

4. de Freitas MTS, Püschel VAA. Heart failure: expressions of personal knowledge about the disease. Rev. esc. enferm. USP. [Internet] 2013;47(4) [acesso em 05 fev 2016]. Disponível: http://dx.doi.org/10.1590/S0080623420130000400021.

5. Dal Boni ALM, Martinez JE, Saccomann ICRS. Quality of life of patients undergoing coronary artery bypass grafting. Acta paul. enferm. [Internet] 2013;26(6) [acesso em 02 de dez 2015]. Disponível: http://dx.doi.org/10.1590/ S0103-21002013000600011.

6. Chu SH, Lee WH, Yoo JS, Kim SS, Ko IS, Oh EG, et al. Factors affecting quality of life in Korean patients with chronic heart failure. Jpn J Nurs Sci. [Internet] 2014;11(1) [acesso em 20 jan 2016]. Disponível: http://dx.doi. org/10.1111/jjns.12002.

7. Carvalho VO, Guimarães GV, Carrara D, Bacal F, Bocchi EA. Validation of the Portuguese Version of the Minnesota Living with Heart Failure Questionnaire. Arq. Bras. Cardiol. [Internet] 2009;93(1) [acesso em 08 nov 2015]. Disponível: http://dx.doi.org/10.1590/S0066-782X2009000700008.

8. Saccomann ICR, Cintra FA, Gallani MCBJ. Quality of life in older adults with heart failure: assessment with a 
specific instrument. Acta paul. enferm. [Internet] 2011;24(2) [acesso em 18 dez 2015]. Disponível: http://dx.doi. org/10.1590/S0103-21002011000200004.

9. Pelegrino VM, Dantas RAS, Clark AM. Health-related quality of life determinants in outpatients with heart failure. Rev. Latino-Am. Enfermagem. [Internet] 2011;19(3) [acesso em 02 fev 2016]. Disponível: http://dx.doi. org/10.1590/S0104-11692011000300002.

10. Bocchi EA, Arias A, Verdejo H, Diez M, Gómez E, Castro P, Interamerican Society of Cardiology. The reality of heart failure in Latin America. J Am Coll Cardiol. [Internet] 2013;62(11) [acesso em 05 jan 2016]. Disponível: http:// dx.doi.org/10.1016/j.jacc.2013.06.013.

11. Pelegrino VM, Dantas RAS, Ciol MA, Clark AM, Rossi LA, Simões MV. Health-related quality of life in Brazilian outpatients with Chagas and non-Chagas cardiomyopathy. Heart Lung. [Internet] 2011;40(3) [acesso 01fev 2016]. Disponível: http://dx.doi.org/10.1016/j.hrtlng.2010.05.052.

12. Hoeskstra T, Jaarsma T, van Veldhuisen DJ, Hillege HL, Sanderman R, Lesman-Leegte I. Quality of life and survival in patients with heart failure. Eur J of Heart Fail. [Internet] 2013;15(1) [acesso em 10 dez 2015]. Disponível: http://dx.doi.org/10.1093/eurjhf/hfs148.

13. dos Santos MA, da Cruz DALM, Barbosa RL. Factors associated to sleep pattern in heart failure patients. Rev. esc. enferm. USP. [Internet] 2011;45(5) [acesso em 04 de jan 2016]. Disponível:http://dx.doi.org/10.1590/S008062342011000500011.

14. Aliti GB, Linhares JCC, Linch GFC, Ruschel KB, Rabelo ER. Signs and symptoms in patients with decompensated heart failure: inference of priority nursing diagnoses. Rev. Gaúcha Enferm. [Internet] 2011;32(3) [acesso em 20 jan 2016]. Disponível: http://dx.doi.org/10.1590/S1983-14472011000300022.

15. dos Santos ACS, do Espírito SantoFH, PestanaL, Daher DV, Santana R. Heart failure: strategies used by elders in search for quality of life. Rev. bras. enferm. [Internet] 2011;64(5) [acesso em 03 jan 2016]. Disponível: http:// dx.doi.org/10.1590/S0034-71672011000500009.

16. Pena FM, Amorim A, Fassbender C, de Oliveira RFJ, de Faria CAC. Insuficiencia cardíaca y depresión: una asociación con resultados negativos. Insuf. card. [Internet] 2011;6(4) [acesso em 05 fev 2016]. Disponível: http:// www.scielo.org.ar/scielo.php?script=sci_arttext\&pid=S1852-38622011000400004.

17. Olano-Lizarraga M, Oroviogoicoechea C, Errasti-Ibarrondo B, Saracíbar-Razquin M.The personal experience of living with chronic heart failure: a qualitative meta-synthesis of the literature. J Clin Nurs. [Internet] 2016;25(1718) [acesso em 15 jan 2016]. Disponível: http://dx.doi.org/10.1111/jocn.13285.

18. Rabelo ER, Aliti GB, Linch GFC, Sauer JM, de Mello AMFS, Martins SM, et al. Non-pharmacological management of patients with decompensated heart failure: a multicenter study - EMBRACE. Acta paul. enferm. [Internet] 2012;25(5) [acesso em 10 jan 2016]. Disponível: http://dx.doi.org/10.1590/S0103-21002012000500003.

19. Malachias MVB, Souza WKSB, Plavnik FL, Rodrigues CIS, Brandão AA, Neves MFT, et al. Sociedade Brasileira de Cardiologia. $7^{a}$ Diretriz Brasileira de Hipertensão Arterial. Arq. Bras. Cardiol. [Internet] 2016;107(3 Suppl 3) [acesso em 05 jun 2016]. Disponível: http://publicacoes.cardiol.br/2014/diretrizes/2016/05_HIPERTENSAO_ ARTERIAL.pdf.

20. Lambrinou E, Kalogirou F, Lamnisos D, Sourtzi P. Effectiveness of heart failure management programmes with nurse-led discharge planning in reducing readmissions: a systematic review and meta-analysis. Int J Nurs Stud. [Internet] 2012;49(5) [acessoem 17 jan 2016]. Disponível: http://dx.doi.org/10.1016/j.ijnurstu.2011.11.002. 